Research Article

\title{
Nasal Carriage and Methicillin Resistance of Staphylococcus aureus among Schoolchildren in Sana'a City, Yemen
}

\author{
Arwa Mohammed Othman (D), Belques Sharaf Al-Huraibi, Rowa Mohammed Assayaghi, \\ and Huda Zaid Al-Shami
}

Microbiology and Immunology Department, Faculty of Medicine and Health Sciences, Sana'a University, Sana'a, Yemen

Correspondence should be addressed to Arwa Mohammed Othman; arwaothman@hotmail.com

Received 4 February 2021; Revised 11 April 2021; Accepted 28 April 2021; Published 7 May 2021

Academic Editor: Joseph Falkinham

Copyright (c) 2021 Arwa Mohammed Othman et al. This is an open access article distributed under the Creative Commons Attribution License, which permits unrestricted use, distribution, and reproduction in any medium, provided the original work is properly cited.

\begin{abstract}
Background. Staphylococcus aureus (S. aureus) is a frequent cause of serious health problems with high morbidity and mortality. The risk of $S$. aureus infections is increased with the emergence of methicillin-resistant $S$. aureus (MRSA). This study aims to determine the nasal carriage rate of both S. aureus and MRSA among schoolchildren in Sana'a city. Methods. This is a crosssectional study conducted from January 2018 to May 2020. Five hundred and thirty-six students were enrolled. Their age ranged from 5 to 19 years with the mean age and standard deviation equal to $13.3 \pm 3.5$ years. Nasal swabs were collected from each student for culturing and methicillin susceptibility testing. Results. Students with positive culture were $271(51 \%)$ males and 265 (49\%) females. S. aureus was isolated from 129 (24\%) students whereas the overall prevalence of MRSA was 8 (1.5\%). S. aureus was significantly recovered from students at the age group of $10-14$ years $\left(\chi^{2}=7.02 ; p=0.03\right)$, females than males $(\mathrm{OR}=1.96$; $\left.\chi^{2}=10.75 ; p=0.001\right)$, and students who were admitted into hospitals $\left(\mathrm{OR}=1.6 ; \chi^{2}=4.89 ; p=0.03\right)$. Nevertheless, there were no significant differences between MRSA carriage and students' age $\left(\chi^{2}=2.3 ; p=0.32\right)$, gender $\left(\mathrm{OR}=1.02 ; \chi^{2}=0.001 ; p=0.63\right)$, and hospital admission $\left(\mathrm{OR}=1.4 ; \chi^{2}=0.25 ; p=0.62\right)$. Conclusions. The prevalence of MRSA is low among schoolchildren in Sana'a city. Age, gender, and previous hospital admission were statistically associated with nasal carriage of $S$. aureus but not MRSA nasal carriage.
\end{abstract}

\section{Background}

Staphylococcus aureus (S. aureus) is a Gram-positive, roundshaped, catalase- and coagulase-positive bacterium. It is a frequent cause of serious health problems with high morbidity and mortality. $S$. aureus colonizes the skin and nasal mucosa and thus can also be considered as normal flora. The anterior nares appear as the main reservoir site for $S$. aureus replication and spread to other body sites. Approximately $25-30 \%$ of healthy individuals are nasal carriers for S. aureus $[1,2]$. Any damage to the epithelial barriers such as trauma and medical or surgical interventions can lead to tissue invasion by $S$. aureus $[3,4]$.

$S$. aureus expresses many virulence factors, and its resistance to antibiotics has gradually increased in recent years. Overuse and/or misuse of antibiotics led to the appearance of strains of $S$. aureus that are resistant to many currently used antibiotics. Penicillin-resistant $S$. aureus produces penicillinase, which hydrolyzes the $\beta$-lactam ring of penicillin leading to penicillin resistance. Later on, scientists developed novel semisynthetic penicillin called methicillin, which is resistant to staphylococcal $\beta$-lactamase $[5,6]$. Methicillin successfully controlled the infection caused by penicillin-resistant $S$. aureus strains. Nevertheless, only 2 years later after methicillin use in treatment, isolation of methicillin-resistant $S$. aureus (MRSA) strains was reported. Methicillin resistance is due to the acquisition of the methicillin resistance $(\mathrm{mec} A)$ gene integrated into the chromosomal element. The mecA gene encodes an altered penicillin-binding protein $2 \mathrm{a}$ or 2' (PBP2a or $\mathrm{PBP} 2^{\prime}$ ) which permits $S$. aureus to grow in the presence of methicillin and other $\beta$-lactam antibiotics [7]. 
MRSA strains have spread worldwide and they cause numerous nosocomial outbreaks in both hospitals and communities. MRSA carriers are predisposed to skin infections, wound infections, bone and joint infections, pneumonia, septicemia, endocarditis, and occasionally toxic shock syndrome. Infections from either hospital-acquired MRSA (HA-MRSA) or community-acquired MRSA (CAMRSA) have increased the challenge of selecting empirical antimicrobial treatments [8-10]. In healthcare institutions, HA-MRSA can be transmitted between patients, or through the hands, clothes, or equipment of healthcare workers (HCWs), and the environment [11].

Nasal carriage of MRSA among HCWs in Taiz city, Yemen, was $55.7 \%$ which is three times much greater than that among HCWs in Saudi Arabia (18\%) and in Oman (15.1\%)-neighboring countries [12-14]. MRSA prevalence among HCWs was $82.3 \%$ in the Gaza strip, $61.0 \%$ in Iraq, $5.3 \%$ in Iran, $4.1 \%$ in China, and $4.6 \%$ in Europe and the United States [15-19].

There is increasing evidence that CA-MRSA is spreading among healthy persons, especially children [20]. In a study conducted by AL-Haj et al., the nasal carriage rate of $S$. aureus was reported to be $23.1 \%$ among public schoolchildren in Sana'a city, but they did not test the isolated bacteria for methicillin and oxacillin sensitivity [21]. Therefore, this study aimed to determine the nasal carriage rate of both $S$. aureus and MRSA among schoolchildren in Sana'a city.

\section{Methods}

2.1. Study Design and Area. A cross-sectional study was conducted from January 2018 to June 2020. Six primary and secondary schools at Sana'a city were randomly chosen by a cluster sampling method. These schools included four public schools (Al Hussain Bin Ali, Roqaya, Omar Bin Al Khattab, and Khaled Bin Al Waleed) and two private schools (Al Amjad and Al Ilmiyah).

2.2. Study Population. Five hundred and thirty-six students were enrolled in this study. Simple random sampling was used to choose students who would participate in the study.

2.3. Inclusion Criteria. All randomly selected schoolchildren who agreed to participate were included.

2.4. Exclusion Criteria. Students who were on antibiotic therapy for one week before sample collection or those who had ulceration or pus at the nares or skin were excluded. Diabetic students were also excluded.

2.5. Sample Size Determination. Based on a study conducted by Al-Haj et al., who reported the prevalence of $S$. aureus among schoolchildren to be $23.1 \%$ [21], and on Central Statistical Organization at Ministry of Planning and Inter Coop (2005-2006) which stated the number of schoolchildren in Sana'a city to be about 2240000 students, the calculated sample size was 471 students at a confidence level of $99 \%$. However, because many students liked to participate, 536 students were enrolled in this study.

2.6. Specimen Collection and Examinations. Samples were obtained from students by using sterile dry-cotton swabs from anterior nares. The swab was inserted 2-3 centimeters in the nasal cavity and rotated 4-5 times both clockwise and anticlockwise before swab withdrawal. Samples were labeled and transported in Amies transport media to the microbiology laboratory at the Faculty of Medicine and Health Sciences within 5-6 hours. At the microbiology laboratory, nasal swabs were inoculated on mannitol salt agar (HiMedia, India). The inoculated agar plates were incubated at $35-37^{\circ} \mathrm{C}$ for $24-48 \mathrm{~h}$. After incubation, plates were investigated for mannitol-fermenter colonies which appear as yellow colonies on mannitol salt agar. Yellow colonies were subcultured on nutrient agar. Golden-yellow colonies on nutrient agar were further examined by Gram stain, catalase, and coagulase tests. Gram-positive cocci, arranged in grape-like clusters, catalase, and coagulase-positive were recorded as S. aureus $[22,23]$.

2.7. Antibiotic Susceptibility Test. All colonies confirmed to be $S$. aureus were tested for methicillin $(5 \mu \mathrm{g})$ and oxacillin $(1 \mu \mathrm{g})$ susceptibility by the modified Kirby-Bauer disc diffusion method. Using a sterile loop, colonies from nutrient agar which were confirmed to be $S$. aureus were picked up, suspended in sterile saline, and mixed to even turbidity. The turbidity intensity of bacterial suspension was adjusted in comparison with the $0.5 \mathrm{McF}$ arland turbidity standard by adding saline or more bacteria. A sterile cotton swab was dipped into the bacterial suspension. Then, the MuellerHinton agar plate (HiMedia, India) was inoculated by swabbing in three directions to evenly distribute the inoculum and make sure there were no gaps between streaks. $5 \mu \mathrm{g}$ methicillin disc and $1 \mu \mathrm{g}$ oxacillin (HiMedia, India) were applied using a sterile needle to come in contact with the agar surface. Inoculated Muller-Hinton plates were incubated at

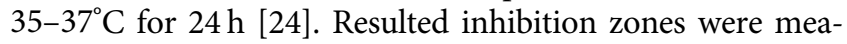
sured using a ruler. Inhibition zone less than or equal to $9 \mathrm{~mm}$ indicated $S$. aureus to be MRSA, while inhibition zones less than or equal to $17 \mathrm{~mm}$ indicated oxacillin-resistant $S$. aureus (Zone Size Interpretative Chart, HiMedia).

2.8. Statistical Analysis. Data analysis was done using SPSS program version 20 (SPSS Inc., Chicago, IL, USA). Descriptive measures (mean \pm standard deviation) were used for quantitative variables. Frequencies and percentages were used to present qualitative variables. Chi-square $\left(\chi^{2}\right)$ test was used for verifying the existence of associations. Probability $(p)$ values $\leq 0.05$ were considered statistically significant.

\section{Results}

Five hundred and thirty-six nasal swabs yielded bacterial growth. The age of the 536 students ranging from 5 to 19 
years with the mean age and standard deviation equal to $13.3 \pm 3.5$ years. They were divided into three age groups: the first age group was from 5 to 9 years, the second age group was from 10 to 14 years, and the third group was from 15 to 19 years. Out of 536, 271 (51\%) students were males and 265 (49\%) students were females. The number of students per class ranged from 3 to 159 students with the mean and standard deviation equal to $71 \pm 34$ students. Four hundred (75\%) students studied at public schools while $136(25 \%)$ studied at private schools (Table 1).

S. aureus was isolated from the anterior nares of 129 (24\%) students. It was recovered more frequently from students at the age group of $10-14$ years $(63,30 \%)$ than from students at the age group of 5-9 years $(22,22 \%)$ and students at the age group of $15-19$ years $(44,19 \%)$. This difference was statistically significant $\left(\chi^{2}=7.02 ; p=0.03\right)$. S. aureus was commonly isolated from females $(80,30 \%)$ than males $(49$, $18 \%)$. The gender difference was statistically significant $\left(\chi^{2}=10.75 ; p=0.001\right)$. S. aureus was recovered from 48 (30\%) students who were admitted into hospitals for either medical treatment or surgery whereas $S$. aureus was found among $81(21 \%)$ students who reported no hospital admission. This difference was statistically significant $\left(\chi^{2}=4.89 ; p=0.03\right)$. Twenty-eight $(21 \%)$ students who were $S$. aureus carries had family members who worked at hospitals or any other health centers compared to 101 (25\%) students who had no family members worked in hospitals or health centers. This difference was statistically nonsignificant $\left(\chi^{2}=1.1 ; p=0.03\right)($ Table 2$)$.

Out of 129 isolated S. aureus, 8 (6.2\%) were resistant to both methicillin and oxacillin discs with an overall prevalence of $1.5 \%$ among school students. Table 3 shows that $3(1.4 \%)$ students in the 10 - to 14 -year age group and 5 (2.2\%) students in the 15- to 19-year age group but no students in the 5- to 9-year age group were carriers for MRSA. This age difference was statistically nonsignificant $\left(\chi^{2}=2.3 ; p=0.32\right)$. MRSA was equally isolated from both males and females $(4,1.5 \%)$ with no significant difference $\left(\chi^{2}=0.001 ; p=0.63\right)$. MRSA was isolated from $3(1.4 \%)$ students who were admitted to hospitals while it was recovered from $5(2.2 \%)$ who were not admitted to hospitals. This difference was statistically nonsignificant $\left(\chi^{2}=2.25 ; p=0.62\right)$. Seven $(1.7 \%)$ students who were MRSA carriers had no family members work at hospitals or health centers, but $1(0.7 \%)$ student who had MRSA at his nares reported a family member works at a health center. This difference was statistically nonsignificant $\left(\chi^{2}=0.69 ; p=0.41\right)($ Table 3$)$.

\section{Discussion}

Nasal carriage of S. aureus is approximately $20-30 \%$ of healthy individuals with high permanent colonization among children [25, 26]. The emergence of MRSA infections has become a worrying problem in the clinical field because MRSA strains are resistant to many antibiotics particularly $\beta$-lactam classes [27]. The purpose of this study was to estimate the nasal carriage rate and methicillin resistance of $S$. aureus among schoolchildren in Sana'a city.
TABLE 1: Sociodemographic features of schoolchildren in Sana'a city, Yemen.

\begin{tabular}{lcc}
\hline & No & $\%$ \\
\hline Age groups of students & & \\
5-9 years & 98 & 18 \\
10-14 years & 210 & 39 \\
15-19 years & 228 & 43 \\
Mean age \pm SD* & $13.3 \pm 3.5$ & \\
\hline Gender & & 51 \\
Males & 271 & 49 \\
Females & 265 & \\
\hline Number of students & & 75 \\
Public schools & 400 & 25 \\
Private schools & 136 & \\
Mean per class \pm SD & $71 \pm 34$ &
\end{tabular}

${ }^{*}$ SD: standard deviation.

TABLE 2: Risk factors for nasal carriage of S. aureus among schoolchildren in Sana'a city, Yemen.

\begin{tabular}{|c|c|c|c|c|c|c|}
\hline & \multicolumn{2}{|c|}{$\begin{array}{l}\text { S. aureus } \\
\text { carriers }\end{array}$} & \multicolumn{2}{|c|}{ Noncarriers } & \multirow[t]{2}{*}{$\chi^{2}$} & \multirow[t]{2}{*}{$p$} \\
\hline & No. & $\%$ & No. & $\%$ & & \\
\hline \multicolumn{7}{|c|}{ Age groups (years) } \\
\hline $5-9$ & 22 & 22 & 76 & 78 & \multirow{3}{*}{7.02} & \multirow{3}{*}{0.03} \\
\hline $10-14$ & 63 & 30 & 147 & 70 & & \\
\hline $15-19$ & 44 & 19 & 184 & 81 & & \\
\hline \multicolumn{7}{|l|}{ Gender } \\
\hline Males & 49 & 18 & 222 & 82 & \multirow{2}{*}{10.75} & \multirow{2}{*}{0.001} \\
\hline Females & 80 & 30 & 185 & 70 & & \\
\hline \multicolumn{7}{|c|}{ History of hospitalization } \\
\hline Yes & 48 & 30 & 110 & 70 & \multirow{2}{*}{4.89} & \multirow{2}{*}{0.03} \\
\hline No & 81 & 21 & 297 & 79 & & \\
\hline \multicolumn{7}{|c|}{ A family member works at hospitals or health centers } \\
\hline Yes & 28 & 21 & 101 & 25 & \multirow{2}{*}{1.09} & \multirow{2}{*}{0.3} \\
\hline No & 107 & 79 & 300 & 75 & & \\
\hline
\end{tabular}

In the present study, the prevalence of $S$. aureus among primary and secondary schoolchildren was $24 \%(129 / 536)$. This finding is consistent with a study conducted by Al-Haj et al., who found the colonization rate of $S$. aureus among public schoolchildren in Sana'a city to be $23.1 \%$ [21]. Our finding was also similar to those reported from other countries [28-30]. However, the nasal carriage rate of $S$. aureus in this study tends to be lower than that reported from Nigeria (56.3\%), India (46.67\%), the United States (39.6\%), the Netherlands (36\%), and Nepal (31\%) [31-35]. On the other hand, the prevalence of $S$. aureus among schoolchildren in our study was higher than that reported from China (5.1\%), Serbia (2.59\%), and Iraq (17.75\%) [36-38]. Variation in the S. aureus nasal carriage from one country to another might be attributed to differences in geographical distribution, sampling, culturing, and diagnostic techniques used by the researchers.

The prevalence of MRSA among schoolchildren in our study was $1.5 \%(8 / 536)$ which was half lower (3\%) than that found by Shetty et al., in Deralakatte, India, who used oxacillin to detect MRAS among schoolchildren [39]. 
TABLE 3: Risk factors for nasal carriage of MRSA among schoolchildren in Sana'a city, Yemen.

\begin{tabular}{|c|c|c|c|c|c|c|}
\hline & \multicolumn{2}{|c|}{ MRSA $^{*}(\text { meth }+ \text { oxa })^{* *}$} & \multicolumn{2}{|c|}{ Other bacteria } & \multirow{2}{*}{$\chi^{2}$} & \multirow{2}{*}{$p$} \\
\hline & No. & $\%$ & No. & $\%$ & & \\
\hline \multicolumn{7}{|c|}{ Age groups (years) } \\
\hline $5-9$ & 0 & 0 & 98 & 100 & \multirow{3}{*}{2.25} & \multirow{3}{*}{0.32} \\
\hline $10-14$ & 3 & 1.4 & 207 & 98.6 & & \\
\hline $15-19$ & 5 & 2.2 & 223 & 97.8 & & \\
\hline \multicolumn{7}{|l|}{ Gender } \\
\hline Males & 4 & 1.5 & 267 & 98.5 & \multirow{2}{*}{0.001} & \multirow{2}{*}{0.63} \\
\hline Females & 4 & 1.5 & 261 & 98.5 & & \\
\hline \multicolumn{7}{|c|}{ History of hospitalization } \\
\hline Yes & 3 & 1.9 & 155 & 98.1 & \multirow[b]{2}{*}{0.25} & \multirow[b]{2}{*}{0.63} \\
\hline No & 5 & 1.3 & 373 & 98.7 & & \\
\hline \multicolumn{7}{|c|}{ A family member works at hospitals or health centers } \\
\hline Yes & 1 & 0.7 & 134 & 99.3 & \multirow{2}{*}{0.69} & \multirow{2}{*}{0.41} \\
\hline No & 7 & 1.7 & 394 & 98.3 & & \\
\hline
\end{tabular}

${ }^{*}$ MRSA: methicillin-resistant $S$. aureus; ${ }^{* *}$ meth: methicillin; oxa: oxacillin.

Moreover, the prevalence of MRSA in this study was much lower than that reported by a study performed in southwest Ethiopia (18.8\%) in which the researchers used cefoxitin $(30 \mu \mathrm{g})$ discs to detect MRSA nasal carriage [40].

Comparing the prevalence of $S$. aureus in our study to the prevalence of $S$. aureus among hospitalized patients at ICU reported by Abdelmonem et al., the prevalence of $S$. aureus among schoolchildren was much less than that among hospitalized patients [12]. In addition, the prevalence of CA-MRSA among schoolchildren in our study was less than the prevalence of HA-MRSA among ICU patients (86.2\% and $100 \%)$ found by Goudarzi et al. and Eftekhar et al., respectively $[41,42]$. This reflects the higher prevalence of HA-MRSA than CA-MRSA.

The current study showed the nasal carriage rate of S. aureus in the 5- to 9-year age group to be $22 \%$ which increased significantly to $30 \%$ in the 10 - to 14 -year age group and then decreased to $19 \%$ in the 15 - to 19 -year age group. Our finding is in agreement with a study conducted by Esposito et al., who reported nasal carriage of $S$. aureus decreases while oropharyngeal carriage increases with age [43]. Decreased nasal carriage in older students may be attributed to their well-developed and stronger immune system.

Concerning gender, our study revealed a significantly higher prevalence of $S$. aureus among females than males but no difference between nasal carriage of MRSA among females and males. Al-Haj et al. reported a higher nasal carriage among females than males, but the difference was nonsignificant [21]. Moreover, Okwu et al. found nasal carriage of $S$. aureus and MRSA to be nonsignificantly higher among females than males [44]. Nevertheless, many studies conducted on schoolchildren found the frequency of $S$. aureus and MRSA nasal carriage to be nonsignificantly higher in males than in females [35, 42, 45]. Tigabu et al. described a higher prevalence of $S$. aureus among males than females but a slightly higher MRSA prevalence among females than males [30]. Statistically significant nasal colonization of $S$. aureus among females than males in our study might be due to wearing a veil among females as an obligatory custom. Wearing a veil might make the anterior nares environment warmer and more humid which in turn may favor $S$. aureus colonization.

Regarding hospitalization, this study showed a highly significant association between nasal colonization of S. aureus with hospitalization; however, nasal carriage of MRSA among schoolchildren showed a nonsignificant association with hospitalization. Nevertheless, no association was found between the presence of first-degree relatives who worked in hospitals or health centers and nasal colonization with S. aureus and MRSA. Our study is in disagreement with the study conducted in Argentina by Gardella et al., who reported no statistical difference between nasal carriage of S. aureus and hospitalization [46]. However, our study is consistent with a study performed in Ethiopia by Reta et al., who reported no association between MRSA prevalence and hospitalization [45]. This finding might imply $S$. aureus acquisition during hospital admission. On the other hand, the low frequency of isolated MRSA makes it difficult to conclude whether MRSA is HA-MRSA or CA-MRSA.

\section{Conclusions}

In conclusion, the prevalence of MRSA among schoolchildren in Sana'a city is low. Age, gender, and previous hospital admission were statistically associated with nasal carriage of $S$. aureus but not with MRSA nasal carriage.

\section{Data Availability}

The data that support the findings of this study are available. Anyone interested can get upon reasonable request from the corresponding author.

\section{Conflicts of Interest}

The authors declare that they have no competing interests.

\section{Acknowledgments}

The authors are grateful to all students and teachers who participated in this study. 


\section{References}

[1] S. Esposito, S. Noviello, and S. Leone, "Epidemiology and microbiology of skin and soft tissue infections," Current Opinion in Infectious Diseases, vol. 29, no. 2, pp. 109-115, 2016.

[2] A. Sakr, F. Brégeon, J. L. Mège, J. M. Rolain, and O. Blin, "Staphylococcus aureus nasal colonization: an update on mechanisms, epidemiology, risk factors, and subsequent infections," Frontiers in Microbiology, vol. 9, p. 2419, 2018.

[3] C. Peña, N. Fernández-Sabe, M. A. Domínguez et al., "Staphylococcus aureus nasal carriage in patients on haemodialysis: role of cutaneous colonization," Journal of Hospital Infection, vol. 58, no. 1, pp. 20-27, 2004.

[4] C. Marshall and E. McBryde, "The role of Staphylococcus aureus carriage in the pathogenesis of bloodstream infection," BMC Research Notes, vol. 7, p. 428, 2014.

[5] C. Rayner and W. J. Munckhof, "Antibiotics currently used in the treatment of infections caused by Staphylococcus aureus," Internal Medicine Journal, vol. 35, pp. S3-S16, 2005.

[6] S. Khoshnood, M. Heidary, A. Asadi et al., "A review on mechanism of action, resistance, synergism, and clinical implications of mupirocin against Staphylococcus aureus," Biomedicine \& Pharmacotherapy, vol. 109, pp. 1809-1818, 2019.

[7] R. H. Schulte and E. Munson, "Staphylococcus aureus resistance patterns in Wisconsin: 2018 surveillance of Wisconsin organisms for trends in antimicrobial resistance and epidemiology (SWOTARE) program report," Clinical Medicine and Research, vol. 17, no. 3, pp. 72-81, 2019.

[8] R. D. Mistry, "Skin and soft tissue infections in ambulatory care settings: setting a new trend," Clinical Infectious Diseases, vol. 70, no. 12, pp. 2719-2720, 2020.

[9] Y. Guo, G. Song, M. Sun, J. Wang, and Y. Wang, "Prevalence and therapies of antibiotic-resistance in Staphylococcus aureus," Frontiers in Cellular and Infection Microbiology, vol. 10, p. 107, 2020.

[10] A. Mairi, A. Touati, and J. P. Lavigne, "Methicillin-resistant Staphylococcus aureus ST80 clone: a systematic review," Toxins (Basel), vol. 12, no. 2, p. 119, 2020.

[11] D. K. Henderson, "Managing methicillin-resistant Staphylococci: a paradigm for preventing nosocomial transmission of resistant organisms," The American Journal of Medicine, vol. 119 , no. 6, pp. S45-S52, 2006

[12] M. O. Abdelmonem, "Nasal carriage of Staphylococcus aureus among healthcare workers in althawra hospital, Taiz city, Republic of Yemen," Australian Journal of Basic and Applied Sciences, vol. 6, no. 7, pp. 417-424, 2012.

[13] O. S. Al-Humaidan, T. A. El-Kersh, and R. A. Al-Akeel, "Risk factors of nasal carriage of Staphylococcus aureus and methicillin-resistant Staphylococcus aureus among health care staff in a teaching hospital in central Saudi Arabia," Saudi Medical Journal, vol. 36, no. 9, pp. 1084-1090, 2015.

[14] N. A. Pathare, H. Asogan, S. Tejani et al., "Prevalence of methicillin resistant Staphylococcus aureus [MRSA] colonization or carriage among health-care workers," Journal of Infection and Public Health, vol. 9, no. 5, pp. 571-576, 2016.

[15] N. A. El Aila, N. A. Al Laham, and B. M. Ayesh, "Nasal carriage of methicillin resistant Staphylococcus aureus among health care workers at Al Shifa hospital in Gaza strip," BMC Infectious Diseases, vol. 17, no. 1, p. 28, 2017.

[16] N. R. Hussein, M. S. Assafi, and T. Ijaz, "Methicillin-resistant Staphylococcus aureus nasal colonisation amongst healthcare workers in Kurdistan Region, Iraq," Journal of Global Antimicrobial Resistance, vol. 9, pp. 78-81, 2017.

[17] M. Askarian, A. Zeinalzadeh, A. Japoni, A. Alborzi, and Z. A. Memish, "Prevalence of nasal carriage of methicillinresistant Staphylococcus aureus and its antibiotic susceptibility pattern in healthcare workers at Namazi hospital, Shiraz, Iran," International Journal of Infectious Diseases, vol. 13, no. 5, pp. e241-e247, 2009.

[18] Y. Kong, J. Ye, W. Zhou et al., "Prevalence of methicillinresistant Staphylococcus aureus colonisation among healthcare workers at a tertiary care hospital in southeastern China," Journal of Global Antimicrobial Resistance, vol. 15, pp. 256261, 2018.

[19] M. Dulon, C. Peters, A. Schablon et al., "MRSA carriage among healthcare workers in non-outbreak settings in Europe and the United States: a systematic review," BMC Infectious Diseases, vol. 14, no. 1, p. 363, 2014.

[20] A. Pathak, Y. Marothi, R. V Iyer et al., "Nasal carriage and antimicrobial susceptibility of Staphylococcus aureus in healthy preschool children in Ujjain, India," BMC Pediatrics, vol. 10, no. 100, 2010.

[21] N. A. Al-Haj, J. M. Hauter, N. H. Al-Bulili, R. A. Al-Hotami, and M. T. Al-Horaibi, "Nasal carriage of Staphylococcus aureus among students of public schools in Sana'a, Yemen," Research Journal of Microbiology, vol. 13, no. 1, pp. 65-69, 2017.

[22] D. F. J. Brown, D. I. Edwards, P. M. Hawkey et al., "Guidelines for the laboratory diagnosis and susceptibility testing of methicillin-resistant Staphylococcus aureus (MRSA)," Journal of Antimicrobial Chemotherapy, vol. 56, no. 6, pp. 1000-1018, 2005.

[23] B. A. Forbes, D. F. Sahm, and A. S. Weissfeld, Bailey and Scott's Diagnostic Microbiology, Mosby Inc., St. Louis, MI, USA, 13th edition, 2013.

[24] Eucast, Disk Diffusion Test Methodology, Eucast, Växjö, Sweden, 2016, https://www.eucast.org/ast_of_bacteria/disk_ diffusion_methodology/.

[25] C. Paulino, R. D. Garcia, and S. Ong, "Staphylococcus aureus nasal carriage rates among children between one-to-five years in Barangay Pio Del Pilar, Makati City," Pediatric Infectious Disease Society of the Philippines Journal, vol. 14, no. 1, pp. 24-33, 2013.

[26] F. Davoodabadi, S. Mobasherizadeh, K. Mostafavizadeh et al., "Nasal colonization in children with community acquired methicillin-resistant Staphylococcus aureus," Advanced Biomedical Research, vol. 5, p. 86, 2016.

[27] R. J. Fair and Y. Tor, "Antibiotics and bacterial resistance in the 21st century," Perspective in Medicinal Chemistry, vol. 6, pp. 25-64, 2014.

[28] D. Eibach, M. Nagel, B. Hogan et al., "Nasal carriage of Staphylococcus aureus among children in the ashanti region of Ghana," PLoS One, vol. 12, no. 1, Article ID e0170320, 2017.

[29] K. Laub, A. Tóthpál, S. Kardos, and O. Dobay, "Epidemiology and antibiotic sensitivity of Staphylococcus aureus nasal carriage in children in Hungary," Acta Microbiologica et Immunologica Hungarica, vol. 64, no. 1, pp. 51-62, 2017.

[30] A. Tigabu, M. Tiruneh, and F. Mekonnen, "Nasal carriage rate, Antimicrobial susceptibility pattern, and associated factors of Staphylococcus aureus with special emphasis on MRSA among urban and rural elementary school children in Gondar, Northwest Ethiopia: a comparative cross-sectional study," Advances in Preventive Medicine, vol. 2018, Article ID 9364757, 11 pages, 2018. 
[31] D. Bogaert, A. van Belkum, M. Sluijter et al., "Colonisation by Streptococcus pneumoniae and Staphylococcus aureus in healthy children," The Lancet, vol. 363, no. 9424, pp. 1871-1872, 2004.

[32] M. J. Kuehnert, D. Kruszon-Moran, H. A. Hill et al., "Prevalence ofStaphylococcus aureus nasal colonization in the United States, 2001-2002," The Journal of Infectious Diseases, vol. 193, no. 2, pp. 172-179, 2006.

[33] K. R. Rijal, N. Pahari, B. K Shrestha et al., "Prevalence of methicillin resistant Staphylococcus aureus in school children of Pokhara," Nepal Medical College Journal: NMCJ, vol. 10, no. 3, pp. 192-195, 2008.

[34] C. Nsofor, V. Nwokenkwo, and C. Nwaokpa, "Nasal carriage of Staphylococcus aureus among apparently healthy school children in Owerri metropolis, Nigeria," Cell Science and Report, vol. 1, no. 2, pp. 117-120, 2015.

[35] A. Singh, L. Agarwal, A. Kumar, C. Sengupta, and R. Singh, "Prevalence of nasal colonization of methicillin-resistant Staphylococcus aureus among schoolchildren of Barabanki district, Uttar Pradesh, India," Journal of Family Medicine and Primary Care, vol. 7, no. 1, pp. 162-166, 2018.

[36] M. Dinic, S. Vukovic, B. Kocic, D. S. Dordevic, and M. Bogdanovic, "Nasal carriage of Staphylococcus aureus in healthy adults and in school children," Acta Facultatis Medicae Naissensis, vol. 30, no. 1, pp. 31-36, 2013.

[37] N. R. Hussein, Z. Basharat, A. H. Muhammed, and S. A. AlDabbagh, "Comparative evaluation of MRSA nasal colonization epidemiology in the urban and rural secondary school community of Kurdistan, Iraq," PLoS One, vol. 10, no. 5, Article ID e0124920, 2015.

[38] Z. Gong, M. Shu, Q. Xia et al., "Staphylococcus aureus nasal carriage and its antibiotic resistance profiles in children in high altitude areas of Southwestern China," Archivos argentinos de pediatria, vol. 115, no. 3, pp. 274-277, 2017.

[39] V. Shetty, K. Trumbull, A. Hegde et al., "Prevalence of community-acquired methicillin-resistant Staphylococcus aureus nasal colonization among children," Journal of Clinical and Diagnostic Research: JCDR, vol. 8, no. 12, pp. DC12DC15, 2014.

[40] T. Kejela and K. Bacha, "Prevalence and antibiotic susceptibility pattern of methicillin-resistant Staphylococcus aureus (MRSA) among primary school children and prisoners in Jimma Town, Southwest Ethiopia," Annals of Clinical Microbiology and Antimicrobials, vol. 12, no. 1, p. 11, 2013.

[41] F. Eftekhar, R. Rezaee, M. Azad, A. Hi, H. Goudarzi, and M. Goudarzi, "Distribution of adhesion and toxin genes in Staphylococcus aureus strains recovered from hospitalized patients admitted to the ICU," Archives of Pediatric Infectious Diseases, vol. 5, no. 1, Article ID e39349, 2017.

[42] M. Goudarzi, S. Seyedjavadi, M. Azad, H. Goudarzi, and H. Azimi, "Distribution of spa types, integrons and associated gene cassettes in Staphylococcus aureus strains isolated from intensive care units of hospitals in tehran, Iran," Archives of Clinical Infectious Diseases, vol. 11, no. 4, Article ID e38813, 2016.

[43] S. Esposito, L. Terranova, A. Zampiero et al., "Oropharyngeal and nasal Staphylococcus aureus carriage by healthy children," BMC Infectious Diseases, vol. 14, p. 723, 2014.

[44] M. Okwu, S. Bamgbala, and W. Aborisade, "Prevalence of nasal carriage of community associated methicillin resistant Staphylococcus aureus (CA-MRSA) among healthy primary school children in Okada, Nigeria," Journal of Natural Sciences Research, vol. 2, no. 4, pp. 61-65, 2012.
[45] A. Reta, L. Gedefaw, T. Sewunet, and G. Beyene, "Nasal carriage, risk factors and antimicrobial susceptibility pattern of methicillin resistant Staphylococcus aureus among school children in Ethiopia," Medical Microbiology and Diagnosis, vol. 4, p. 177, 2014.

[46] N. Gardella, S. Murzicato, S. Di Gregorio et al., "Prevalence and characterization of methicillin-resistant Staphylococcus aureus among healthy children in a city of Argentina," Infection, Genetics and Evolution, vol. 11, no. 5, pp. 1066-1071, 2011. 